\title{
Successive reductions of liquid and solid sucrose rewards
}

\author{
R. A. BURNS, E. S. DUPREE, and T. S. LORIG \\ Georgia Southwestern College, Americus, Georgia 31709
}

\begin{abstract}
Successive reductions in sucrose rewards are examined in two analogous runway experiments. In Experiment 1, rats were trained for 24 preshift days with $1 \mathrm{ml}$ of either $30 \%$ or $3 \%$ sucrose solution as reward. Training trials were administered two per day with an intertrial interval (ITI) of 3-5 min. During 2 postshift days, all animals received 20 trials (10 per day with the same ITI) with the $3 \%$ reward. Experiment 2 employed essentially the same procedure, except the rewards were either eight or two Noyes sucrose $(92.4 \%)$ pellets in preshift. Postshift was to the two-pellet reward. The data suggested a successive negative contrast effect (SuNCE) in Experiment 2 with solid pellets, but not in Experiment 1 with liquid solutions. The findings are briefly considered in light of the sequential and frustration accounts of the SuNCE.
\end{abstract}

In the runway, a downshift in magnitude of reward typically produces the successive negative contrast effect (SuNCE), slower running by a downshifted group than an unshifted small-reward control (e.g., Crespi, 1942; Elliott, 1928). The SuNCE does not, however, occur with reductions in sucrose rewards (e.g., Homzie \& Ross, 1962; Rosen, 1966).

One account of the SuNCE is derived from sequential learning theory (Capaldi \& Lynch, 1967). Each reward magnitude is assumed to produce distinct traces or aftereffects that become conditioned to the instrumental response by reward on the subsequent trial. By this view, the SuNCE is the result of generalization decrement produced by the change in the sensory consequences of responding.

\section{EXPERIMENT 1}

The design of the present experiment was encouraged by the evaluation (Burns, 1976) of a hypothesis, based on sequential learning theory, that would account for the failure to obtain the SuNCE with sucrose rewards. To the extent that the hypothesis is based upon those sequential principles that are proposed to account for the SuNCE when it occurs, this experiment seeks to examine the feasibility of the generalization-decrement account of contrast effects in general.

Following sequential theory, the lack of the SuNCE could be due to the ineffective conditioning of reward aftereffects. If sucrose reward aftereffects were nondifferentiable for some reason, then generalization decrement would not be expected after a downshift

This work was supported by the Charles L. Mix Memorial Fund through the Department of Psychology at Georgia Southwestern College. Requests for reprints should be sent to R. A. Burns, Department of Psychology, Georgia Southwestern College, Americus, Georgia 31709. in sucrose (Capaldi, 1967; Flaherty, Riley, \& Spear, 1973; Likely, Little, \& Mackintosh, 1971; Mackintosh, 1974). In a pair of experiments examining sequential learning in paradigms other than the contrast paradigm, Burns (1976) showed that sucrose traces are clearly differentiated under a restricted set of conditioning parameters, two trials per day with a 3- to 5-min intertrial interval (ITI), a result that implies that the SuNCE would occur with sucrose under the proper training conditions. Yet a shift from $30 \%$ sucrose to $3 \%$ sucrose failed to produce the SuNCE following training under these optimal aftereffect conditioning parameters (Burns \& Burns, 1978). The postshift procedure of that sucrose-shift experiment did not, however, involve intentional confounding of the number of daily trials with changes in reward magnitude, but such a procedure is common in reward-sequence experiments involving shifts (e.g., Leonard, 1969). To examine the possibility that this confounding might somehow potentiate a generalization-mediated SuNCE, rats in the present experiment were shifted from $30 \%$ to $3 \%$ sucrose solution following preshift training with 2 trials per day and a 3- to 5-min ITI; during a postshift phase, all animals received the $3 \%$ solution on each of 10 daily trials.

\section{Method}

Subjects. The subjects were 16 experimentally naive male Dublin Sprague-Dawley rats obtained from Flow Laboratories. The rats were approximately 90 days old at the beginning of preliminary training.

Apparatus. The apparatus, fully described elsewhere (Burns, 1976), was a black wooden runway with a white goalbox positioned at a 90-deg angle. Photocell logic to two Standard Electric timers allowed recording of start and run times.

Preliminary training. All animals were housed in individual living cages and reduced to $85 \%$ of their free-feeding body weight. On 2 successive days, following random assignment of rats to two groups $(n=8)$, animals were allowed $15 \mathrm{~min}$ free access to either $30 \%$ or $3 \%$ sucrose solution (mixed in tap water 
by weight) administered on a walled exercise table. All animals were then randomly assigned to one of four squads $(n=4)$, and goalbox placements, which entailed placing each rat in the goalbox containing $1 \mathrm{ml}$ of the appropriate sucrose solution, were administered twice a day for 4 days. During the entire experiment, the order of running of squads and rats within squads was determined randomly each day, and for goalbox and preshift training, the concentration of sucrose for each rat was the same as during the table feedings (30\% or $3 \%$ ).

Preshift training. There were 24 preshift days, two trials each day. A trial began with the opening of the startbox door $3 \mathrm{sec}$ after the rat was placed in the startbox. When the animal entered the goalbox, the retrace door was closed, and the rat was removed after the reward $(1 \mathrm{ml}$ of $30 \%$ or $3 \%$ sucrose delivered in a goal cup fashioned from a teaspoon) was consumed. Throughout the experiment, subjects were run in rotation within a squad to maintain an ITI of 3-5 min. Approximately $15 \mathrm{~min}$ after each daily running, each rat was weighed and fed its calculated ration in the living cage.

Postshift training. Following the 24 preshift days, all $30 \%$ animals were shifted to the equal volume of $3 \%$ solution for the 20 trials of postshift training. Trials were administered 10 per day over a 4-day period, with a random half of the animals run on the first and third days and the remaining animals on the second and fourth days.

\section{Results}

Raw time scores were transformed $[10 \cdot \ln (x+1)]$ for analysis. The preshift training produced a character-

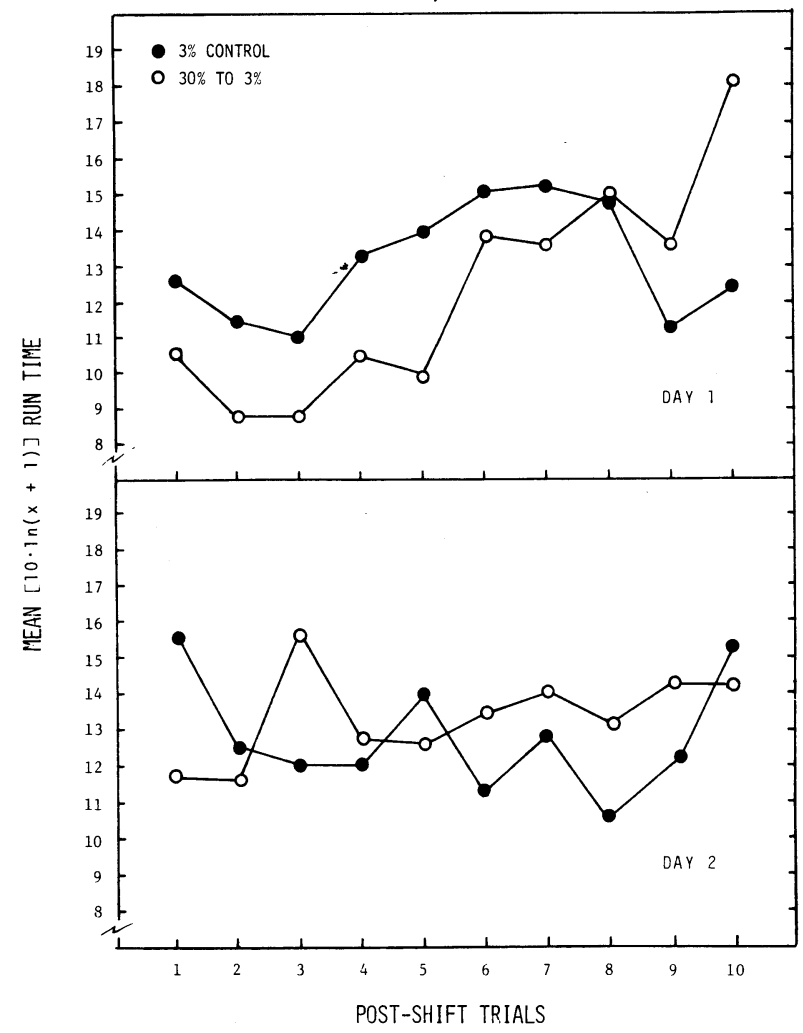

Figure 1. Mean transformed run times for Day 1 (top panel) and Day 2 (bottom panel) of postshift. Each point is a trial mean for either the downshifted group (open circles) or the unshifted control (closed circles). istic between-groups reward magnitude discrimination. The $30 \%$ group ran faster than the $3 \%$ group $[\mathrm{F}(1,14)$ $=6.77, \mathrm{p}<.05]$. Repeated-measures analysis of variance during postshift showed nonsignificant interactions of Groups by Trials $[\mathrm{F}(9,126)=1.62, \mathrm{p}>.05]$ and Groups by Days by Trials $[F(9,126)=1.72, p>.05]$. The overall effect of trials, however, was significant, as well as the Days by Trials interaction $[F(9,126)=2.15$, $p<.05 ; F(9,126)=2.33, p<.05]$. Figure 1 shows the mean transformed run times over trials for each of the postshift days. Separate analyses on each day showed a significant effect of trials on Day $1[F(9,126)=3.44$, $\mathrm{p}<.05]$, but not on Day $2(\mathrm{~F}<1)$, suggesting that the increased number of daily trials from pre- to postshift produced some early disruption in both groups; neither of the interactions shown in Figure 1 was significant $[F(9,126)=1.91, p>.05 ; F(9,126)=1.31, p>.05]$. The mean for the shifted group on Trial 10 of Day 1 was greater than the control mean $[1 \mathrm{sd}(140)=4.55$, $\mathrm{p}<.05]$, but no other between-groups trial means differed.

\section{Discussion}

The postshift conditions ended the preshift reward discrimination, unlike the earlier experiment, in which postshift trials were administered at 2 rather than 10 per day (Burns \& Burns, 1978), but no clear SuNCE developed. The last trial of Day 1 in postshift could suggest contrast, but the lack of a Groups by Trials interaction coupled with (1) the results of a subsequent replication in which no crossover at all was observed (Burns, Note 1), and (2) the fact that the Trial 10 mean was unduly influenced by a single extreme score warrant the alternative interpretation.

An argument, weakened by the necessity of betweenexperiments comparisons, could be made against a generalizationdecrement account of the SuNCE based on the results of this experiment since the conditions previously shown to be effective in producing sucrose aftereffect learning in other sequential paradigms (Burns, 1976) were employed here without generating contrast.

\section{EXPERIMENT 2}

The question of why the SuNCE occurs reliably under a variety of conditions with reduction of ordinary foods, such as Noyes pellets (cf. Dunham, 1968), but not with the reduction of sucrose solutions, is, of course, an important one. The SuNCE has had a rich theoretical history. There have been several implications that the critical difference is a liquid-solid or licking-chewing difference. Flaherty et al. (1973), for example, have proposed that licking might reduce the number of stimulus dimensions, relative to chewing, thereby effecting less generalization decrement when reward is reduced. Rosen (1966) suggested that constant licking (of sucrose solution) prevents the conditioning of frustration (Amsel, 1958) to the goalbox cues. Because these two antithetical views predict that a shift in solid sucrose would produce the SuNCE, while numerous studies have failed to obtain the effect with liquid 
sucrose reductions, Experiment 2 employed a successive shift in the number of sucrose pellets.

\section{Method}

Subjects and Apparatus. The subjects were 16 naive male rats of the same age and breeding as those in Experiment 1. The apparatus was also the same.

Training. The preliminary training was the same as in Experiment 1 except that no table feeding was employed, there were 3 rather than 4 days of goalbox placements, and the reward was either eight or two 45-mg Noyes sucrose $(92.4 \%)$ pellets, rather than a $30 \%$ or $3 \%$ solution. There were 20 preshift training days, the procedure for which was the same as in Experiment 1 except for the type of reward (eight or two pellets), and 2 postshift days, on which all animals received 10 trials per day with the two-pellet reward.

\section{Results}

Analysis of variance of the transformed run times for the last 4 days of preshift (during asymptotic acquisition) showed that the eight-pellet reward produced faster run times than the two-pellet reward $[F(1,14)=5.62, p<.05]$ and that running was at asymptote (Days: $F<1$ ) for both groups (Groups by Days: $F<1)$. The postshift analysis showed a main effect of trials $[F(9,126)=2.31, p<.05]$, as in Experiment 1, but the Groups by Trials interaction was significant $[F(9,126)=2.00, p<.05]$. None of the interactions with days was significant. Figure 2 shows the run times over trials for each of the postshift days.

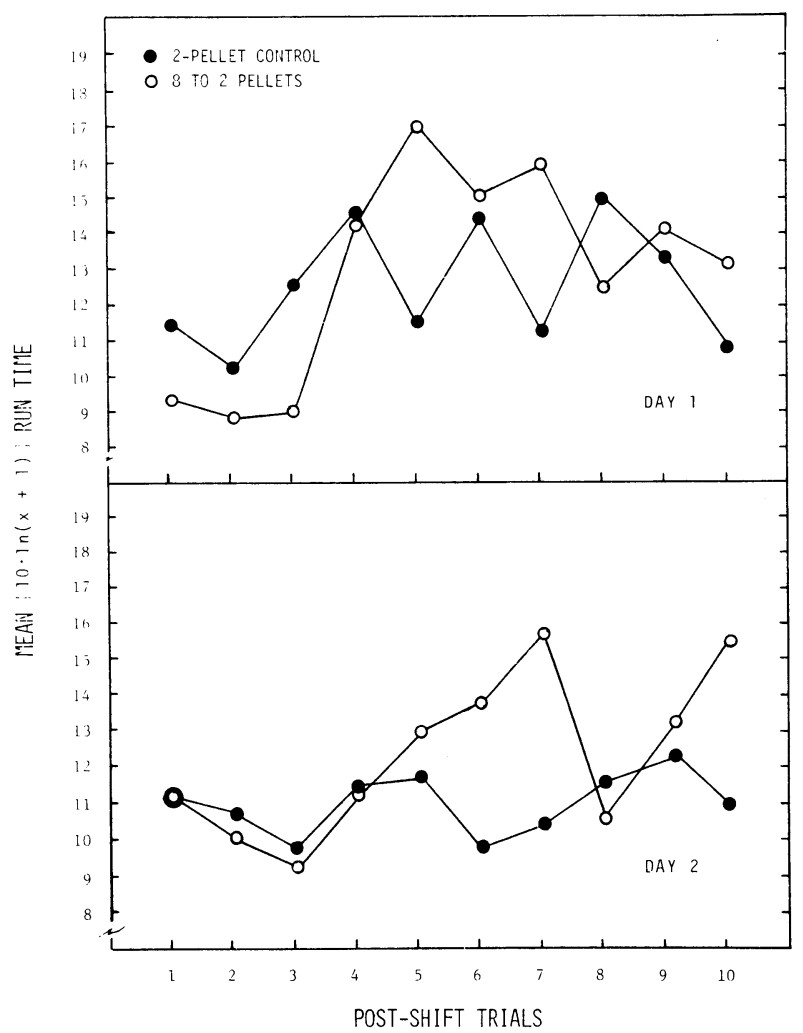
days.

Figure 2. Mean transformed run times for both postshift
Although neither of the interactions shown in Figure 2 was significant $[F(9,126)=1.44, p>.05 ; \quad F<1]$, the shifted animals ran slower than controls on Trial 5 of Day $1[1 \mathrm{sd}(140)=5.41, \mathrm{p}<.05]$ and on Trial 7 of Day $2[\operatorname{lsd}(140)=4.84, p<.05]$. The main effect of trials was again significant on Day $1[\mathrm{~F}(9,126)=2.17$, $\mathrm{p}<.05]$, but not on Day $2[\mathrm{~F}(9,126)=1.01, \mathrm{p}>.05]$.

\section{GENERAL DISCUSSION}

The Groups by Trials postshift interaction in Experiment 2 is strongly suggestive of the SuNCE. The interaction did not appear in Experiment 1. The results of the two experiments considered together emphasize the potential importance of the liquid vs. solid reward distinction as a key to the understanding of the inability of sucrose reward reductions to produce the SuNCE. Experiment 2 showed what is apparently the first clear demonstration of the SuNCE with a single sucrose shift in the runway. Another experiment (Shanab, France, \& Young, 1975) generated a SuNCE with a shift from $1016 \%$ sucrose pellets to a single $4 \%$ pellet, but, unfortunately, the pellets were a mixture of sucrose and other foods; their animals ran faster in preshift for $4 \%$ pellets than for equal numbers of $16 \%$ pellets. The reduction in sucrose, then, was confounded with decreases in food reward. Since pure $(92.4 \%$ ) sucrose pellets were employed in Experiment 2, the confounding did not exist.

The data from Experiment 2 support both the Rosen (1966) hypothesis, based on frustration theory, and the hypothesis of Flaherty et al. (1973), based on sequential theory; however, the general pattern of results casts some doubt on the sequential explanation. Rats readily develop differential responding based on liquid sucrose traces (Burns, 1976), but Experiment 1 shows that the conditions that seem most effective in generating that differential responding do not generate a reliable SuNCE.

\section{REFERENCE NOTE}

1. Burns, R. A. Reward reduction to small reward and nonreward without contrast effects. Paper presented at the annual meeting of the Southern Society for Philosophy and Psychology, Orlando, Florida, 1978.

\section{REFERENCES}

AMSEL, A. The role of frustrative nonreward in noncontinuous reward situations. Psychological Bulletin, 1958, 55, 102-119.

BuRNs, R. A. Effects of sequences of sucrose reward magnitudes with short ITIs in rats. Animal Learning \& Behavior, 1976, 4, 473-479.

Burns, R. A., \& Burns, D. P. Reduction in sucrose reward magnitude without generalization decrement. Bulletin of the Psychonomic Society, 1978, 12, 196-199.

CAPALDI, E. J. A sequential hypothesis of instrumental learning. In K. W. Spence \& J. T. Spence (Eds.), The psychology of learning and motivation (Vol. 1). New York: Academic Press, 1967.

CAPAldi, E. J., \& LYNCh, A. D. Repeated shifts in reward magnitude: Evidence in favor of an associational and absolute (noncontextual) interpretation. Journal of Experimental Psychology, 1967, 75, 226-235.

CRESPI, L. P. Quantitative variation of incentive performance in the white rat. American Journal of Psychology, 1942, 55, 467-517.

DunhaM, P. J. Contrasted conditions of reinforcement: A selective critique. Psychological Bulletin, 1968, 69, 295-315.

Elliotr, M. H. The effect of change of reward on the maze performance of rats. University of California Publications in Psychology, 1928, 4, 19-30. 
Flaherty, C. F., Riley, E. P., \& Spear, N. E. Effects of sucrose concentration and goal units on runway behavior in the rat. Learning and Motivation, 1973, 4, 163-175.

Homzie, M. J., \& Ross, L. E. Runway performance following a reduction in the concentration of a liquid reward. Journal of Comparative and Physiological Psychology, 1962, 55, 1029-1033.

LEONARD, D. W. Amount and sequence of reward in partial and continuous reinforcement. Journal of Comparative and Physiological Psychology, 1969, 67, 204-211.

Likely, D., Little, L., \& Mackintosh, N. J. Extinction as a function of magnitude and percentage of food or sucrose reward. Canadian Journal of Psychology Review of Canadian Psychology, 1971, 25, 130-137.
Mackintosh, N. J. The psychology of animal learning. New York: Academic Press, 1974.

Rosen, A. J. Incentive-shift performance as a function of magnitude and number of sucrose rewards. Journal of Comparative and Physiological Psychology, 1966, 62, 487-490.

Shanab, M. E., France, J., \& Young, T. Negative contrast effect obtained with downshifts in magnitude but not concentration of solid sucrose reward. Bulletin of the Psychonomic Society, 1975, 5, 429-432.

(Received for publication July 24, 1978.) 